\title{
sciendo
}

CIVIL AND ENVIRONMENTAL ENGINEERING REPORTS

E-ISSN 2450-8594

CEER 2020; 30 (1): 196-212

DOI: $10.2478 /$ ceer-2020-0015

Original Research Article

\section{EXPERIMENTAL INVESTIGATION OF CEMENTITIOUS MATERIALS USING THE PRISM TECHNIQUE}

\author{
Rahma MESSAOUDI ${ }^{1}$, Cherif BOUZERIRA ${ }^{2}$, Morad GRIMES ${ }^{3}$, \\ Hocine OUCIEF ${ }^{4}$ \\ ${ }^{1}$ Department of civil engineering, LGCE Laboratory, University of MSB Jijel \\ ${ }^{2}$ Department of civil engineering, NDT Laboratory, University of MSB Jijel \\ ${ }^{3}$ Department of electronics, NDT Laboratory, University of MSB Jijel \\ ${ }^{4}$ Department of civil engineering, MGE Laboratory, University of BM Annaba
}

\begin{abstract}
Numerous studies have shown that the non-destructive testing has proved the exceptional role in supervising the quality of concrete. Today, they represent an alternative that contributes to the resolution of materials diagnostic problems without altering them. This work presents an experimental study using a pulse-echo method that has the advantage of generating two types of wave (compressional and shear) by a mode conversion using just one transducer. The samples were made in a prismatic form. The results recovered from backscattered echoes are extracted using an estimation algorithm. These data were exploited to determine velocities of the tested material then the reflection coefficients for related them with the compressive strength. The tests were conducted with six samples with varied water/cement ratio. The results obtained showed that the prism technique has the potential to evaluate characteristics cementitious material using this proposed process.
\end{abstract}

Keywords: prism technique, cementitious material, compressional wave, shear wave, reflection coefficient

\footnotetext{
${ }^{1}$ Corresponding author: Department of civil engineering, LGCE Laboratory, University of MSB Jijel, Ouled Aissa, BP 98, Jijel 18000, Algeria, e-mail: messaoudirahma@univ-jijel.dz, tel. $+213671139100$
} 


\section{INTRODUCTION}

Nowadays, the majority of the buildings is partially or entirely made from cementitious materials, such as concrete which is composed of aggregates bound by a hydrated cement paste, where the quality and quantity of the latter are among many factors that guarantee the sustainability of the structure. Due to technological advances in the building materials sector, total knowledge of all different properties and behavior still remains incomplete. To bridge the gap that often exists between the evolution of materials and evaluation methods, researchers generally make use of the field of non-destructive ultrasonic testing, which has proved its role and exceptional interest in cementitious materials. However, the reliability of the performance diagnosis, the location of the damage and the possibility of access to the concrete elements contributed to the evolution of this field in civil engineering (NDT-CE). There are several works on non-destructive methods, among them are the acoustic method (Aggelis et al., 2019), physical methods (Brozovsky, 2014) and radar methods (Dinh et al., 2019), Hussain and Akhtar (2017) summarized the history literature about NDT methods. The acoustic method can be applied with three techniques that are acoustic emission, impact-echo and ultrasonic pulse velocity (Payan et al., 2018; Hellier, 2003), the parameter usually measured is ultrasonic pulse velocity (usually abbreviated as UPV). In this context, the UPV of concrete has been the subject of much research. Studies have revealed that it is affected by several factors, including the variation of the aggregates (type, form, quantity, maximum size) (Trtnik et al., 2009; Abo-Qudais, 2005; Uddin and Mahmood, 2016), porosity (Hernandez et al., 2000; Panzera et al., 2008), age and w/c ratio (AboQudais, 2005) and others. In addition, from the UPV mathematical relations and numerical models were conducted to predict the concrete properties (compressive strength, permeability, modulus of elasticity, bulk density ... etc.) (Trtnik et al., 2009; Panzera et al., 2008). Likewise, at an early age, Boumiz et al. (1996) have successfully studied the evolution of the setting process and mechanical properties of cement pastes based on the UPV. In addition, correlations with compressive strength have been made (Al-Mufti and Fried, 2012; Kheder et al., 2003), although there is no theoretical relationship between the compressive strength of concrete and the UPV. Indeed, this was the problem faced by some researchers such as Qasrawi (2000), Turgut and Kucuk (2006), because they did not find a good correlation between the compressive strength and UPV for cement materials. The research concluded that it would be futile to attempt to establish this relationship unless it was only to establish previous relationships with the same type of concrete produced in-situ as that of the laboratory. Bouhadjera and Bouzerira (2005) reports that this is the case, even for homogeneous and linearly elastic materials, it is an intrinsic difficulty. On 
the other hand, although there is a variety of methods that study the hydration of cement, each type to its specificities. There are non-destructive studies that have focused on this age period and they have adopted the principle of ultrasonic pulse reflection measurements at the tested buffer-material interface instead of the UPV. They succeeded in linking the development of the reflection coefficient to the evolution of hydration in cementitious materials and they showed the influence of the w/c ratio and the temperature of the cementitious materials on the development of this coefficient, thus completing the knowledge acquired by existing methods (Voigt, 2005). At an early age, the reflection coefficient seems to be a promising parameter. For this reason, this study attempts to establish a projection of the idea of the monitoring of a cementitious material by this parameter on the curing time. For any study, the choice of nondestructive equipment must meet the criteria of economic aspects, applicability and simplicity (Mix, 2005). Regarding this work, the prism technique will be used, it was designed by A. Bouhadjera (2005). Blitz (1997) reports that nondestructive methods that have a manual mode of operation give us the opportunity to instantly choose decisions during the course of the trial. This possibility is offered by the technique prism method. It is known that the ratio water/cement plays a dominant role on the mechanical strength (Boumiz et al., 1996) and the structuring of the cement paste. Research is generally focused on the quality (or w/c) of the paste and not on its quantity (Hermida, 2008), including this study, which highlights the effect of the variation of the $\mathrm{w} / \mathrm{c}$ ratio on the reflection coefficient. In particular, the evolution of this coefficient during the curing time will be shown, in order to correlate with the mechanical strength of the material to see if it can be a possible alternative to inform us about the prediction of the compressive strength.

\section{MATERIALS AND EXPERIMENTAL PROCEDURE}

\subsection{Specimen, materials and curing conditions}

The experiment was carried out on local white cement pastes (CPJ-CEM II/A $52.5 \mathrm{~N}$ ) commercialized by 'Lafarge'. Cement pastes are prepared using a mortar mixer with a capacity of 5 liters, by mixing the water and the cement for 3 minutes divided into three phases: 90 s at slow speed, 30s downtime and a mix of $90 \mathrm{~s}$ at fast speed. For each formulation, six specimens are manufactured. The test samples are in prismatic form, side and height of $6 \mathrm{~cm}$ whose main section is an isosceles right triangle (Fig. 1). They had six w/c ratios ranging from 0.35 to 0.6 , these mass proportions are water compared to a single proportion of cement. The specimens were maintained for 24 hours in their molds in the laboratory. 
After demoulding, they are kept in water at a temperature of $20^{\circ} \mathrm{C} \pm 2^{\circ}$ until the time of the test.

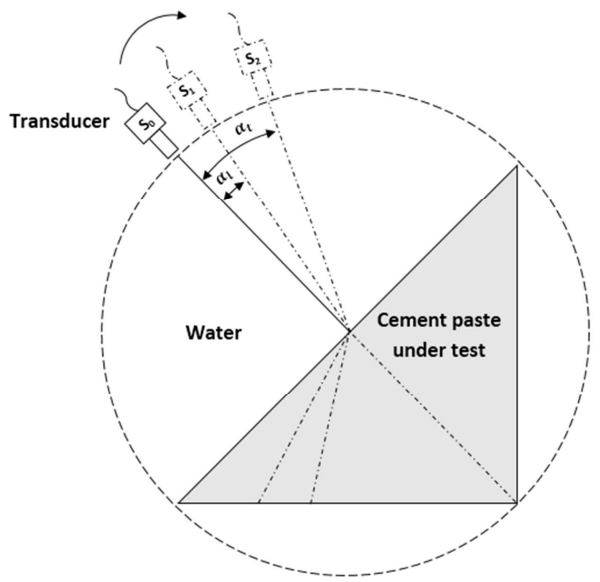

Fig. 1. Prism technique configuration

\subsection{Description of the experimental setup}

An immersion experiment was conducted using the device shown in Fig. 2.

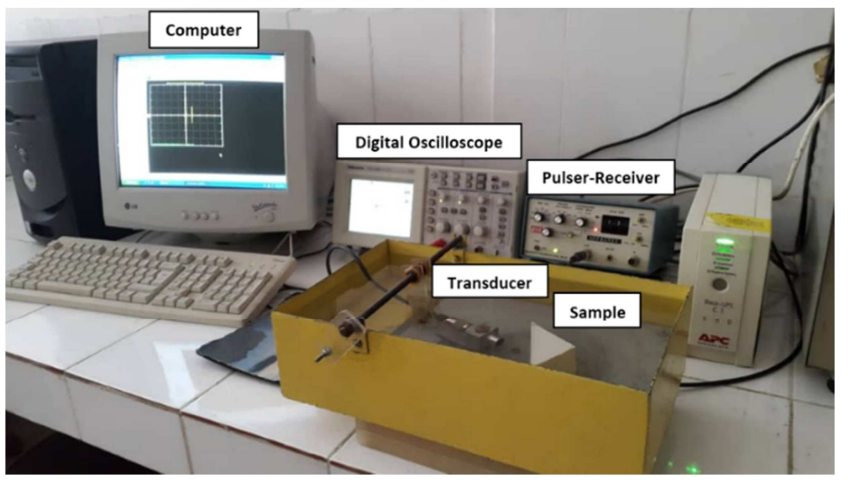

Fig. 2. The experimental test setup

The measuring system consists of a pulse generator with the reference Sofranel (Panametrics, transmitter/receiver, type 5077PR), a piezoelectric transducer Panametrics $(E / R)$ of frequency $1 \mathrm{MHz}$ with a support fixed to the top of a movable ring around a graduated circle with a radius $\mathrm{R}$ (of the order of $4 \mathrm{~cm}$ ), a fixed platform (prism support) and a parallelepiped metal tank filled with water. The transducer is digitized by a digital oscilloscope (Tektronix TDS 1002) to view echoes and a computer with WaveStar software for acquiring and storing signal data as an Excel file (Time, Amplitude). For all specimens, the received signal is amplified using an appropriate gain $(15 \mathrm{~dB})$. 


\subsection{Ultrasonic immersion testing}

First, the measurements are made according to the normal in the center of the hypotenuse of the prism. The reference signal $S_{0}$ (Fig. 3.a) is then obtained when the sample is adjusted parallel to the transducer, it corresponds to the angle $\alpha$ which is equal to zero (normal incidence). Then, by changing the position of the transducer, this angle will be increased until the disappearance of the first echo and the appearance of a second, which is linked to the first angle $\alpha_{C}$ corresponding to the compressional wave, beyond the first critical angle $\alpha_{c r 1}$ the compression mode transmitted no longer propagates. While continuing to increase this angle, the second echo $S_{1}$ (Fig. 3.b) disappears and there appears a third $S_{2}$ (Fig. 3.c) at the second angle $\alpha_{S}$ which corresponds to the shear wave in the sample by a mode conversion. Beyond the second critical angle $\alpha_{c r 2}$, no wave is transmitted in the material means that the incident wave is completely reflected, where it is transformed into a surface wave. The three echoes $S_{0}, S_{1}$ and $S_{2}$ of the cement paste are recorded for different ratio $(w / c)$ since the second day of demoulding with a regular time step of 24 hours. The measurement is made as soon as the highest amplitude mode appears. The estimation of the time of flight (compression or shear) was made by comparing the $S_{0}$ with $S_{1}$ or $S_{2}$, since the latter two containing less information about the water/specimen interface echo.

For each sample, the velocity is given by:

$$
V_{C, S}=a / 2 \times \Delta t_{C, S}
$$

$a$ represents the side of the prism (m) and $\Delta t$ : the time of flight (s).

$V_{C}$ and $V_{S}$ are the compressional and shear velocities in the solid, respectively cited.

The own technical principle of this method is the refractive angle of $45^{\circ}$ within the prism, wherein a total reflection of the incident wave occurs. The wave follows the same path as its path to the sample when it moves towards the transducer. By applying Snell's law, this occurs when the angle of incidence equals: $\alpha_{1}=\arcsin \left(V / \sqrt{2} V_{C}\right)$ for the refracted compressional wave and $\alpha_{2}=\arcsin \left(V / \sqrt{2} V_{S}\right)$ for the shear wave by a mode conversion. Where $V$ is the velocity of the compressional waves in the water which is equal to $1480 \mathrm{~m} / \mathrm{s}$.

The first and second critical angle corresponding to the disappearance of the compressional and shear waves respectively, are given by:

$$
\alpha_{c r 1,2}=\arcsin \left(\frac{V}{V_{C, S}}\right)
$$

Before these data acquisitions can be exploited, it is preferable to improve the accuracy of waveforms in the time domain, In order to obtain a finer 
characterization of the specimens. The recovery of the ultrasound wave arrival time parameter was made with an algorithm known as Space alternating generalized expectation maximization (SAGE). It represents a new approach to the analysis and complete decomposition of waveform properties, the algorithm has been studied and well explained by Grimes et al. (2012). In order to replicate the experimental echo, an initial vector of parameters is needed containing: the width of the distribution $\alpha=4.10^{12}$, the amplitude of the signal envelope $\beta=5 \mathrm{~V}$, the center frequency of pulse is $\mathrm{f}=1 \mathrm{MHz}$, the arrival time of the wave packet group velocity $\tau$, the phase shift of the wave is $\varphi=\pi / 3 \mathrm{rad}$. The experimentally acquired signal and the estimated signal (dashed line) are shown in Fig. 3.

\subsection{Measurement of reflection and transmission coefficient}

The present study is interested in the reflection and transmission energy coefficients (the reflectance and the transmittance) from the water/cement interface. In the liquid/solid case, the reflection coefficient $\mathrm{R}$ and the transmission coefficient D (compressional or shear) of the acoustic pressure are given by Krauträmer, 1990:

$$
\begin{gathered}
R=\frac{1}{N}\left(\left(\frac{V_{S}}{V_{C}}\right)^{2} \sin \left(2 \alpha_{C}\right) \sin \left(\alpha_{S}\right)+\cos ^{2}\left(2 \alpha_{S}\right)-\frac{\rho V}{\rho_{S} V_{C}} \frac{\cos \left(\alpha_{C}\right)}{\cos \left(\alpha_{i}\right)}\right) \\
D_{C C}=\frac{2}{N} \cos \left(2 \alpha_{S}\right) \\
D_{S C}=-\frac{2}{N}\left(\frac{V_{S}}{V_{C}}\right)^{2} \sin \left(2 \alpha_{C}\right)
\end{gathered}
$$

With abbreviation:

$$
N=\left(\frac{V_{S}}{V_{C}}\right)^{2} \sin \left(2 \alpha_{C}\right) \sin \left(2 \alpha_{S}\right)+\cos ^{2}\left(2 \alpha_{S}\right)+\frac{\rho V}{\rho_{S} V_{C}} \frac{\cos \left(\alpha_{C}\right)}{\cos \left(\alpha_{i}\right)}
$$

$\alpha_{i}$ : Angle of incidence wave in the liquid.

$\alpha_{C}, \alpha_{S}$ : Angle of compressional and shear waves in the solid.

$\rho_{s}$ : Density of the solid.

At the liquid/solid interface, the reflection and pressure transmission coefficients can be seen explicitly by the following relationships (David and Cheeke, 2002):

$$
\begin{gathered}
R\left(\alpha_{i}\right)=\frac{Z_{e f f}-Z}{Z_{e f f}+Z} \\
T_{C}\left(\alpha_{i}\right)=\left(\frac{\rho}{\rho_{S}}\right) \frac{2 Z_{L} \cos 2 \alpha_{t}}{Z_{C} \cos ^{2} 2 \alpha_{S}+Z_{S} \sin ^{2} 2 \alpha_{S}+Z} \\
T_{S}\left(\alpha_{i}\right)=-\left(\frac{\rho}{\rho_{S}}\right) \frac{2 Z_{T} \sin 2 \alpha_{t}}{Z_{C} \cos ^{2} 2 \alpha_{S}+Z_{S} \sin ^{2} 2 \alpha_{S}+Z}
\end{gathered}
$$


Where: $Z=\frac{\rho V}{\cos \alpha_{i}}, Z_{C}=\frac{\rho_{S} V_{C}}{\cos \alpha_{C}}, Z_{S}=\frac{\rho_{S} V_{S}}{\cos \alpha_{S}}$

The effective impedance:

$$
Z_{e f f}=Z_{C} \cos ^{2} 2 \alpha_{S}+Z_{S} \sin ^{2} 2 \alpha_{S}
$$

These formulas give the coefficients of reflection and transmission of the pressure. In this present experimental work, the objective is to determine the influence of $w / c$ on this coefficients of acoustic intensity which are of great importance and easier to understand than the pressure ratios as it has been quoted by several authors (David and Cheeke, 2002).

The reflection and transmission coefficients of the acoustic intensity are given by the following formulas according to angle of incidence and reflection (David and Cheeke, 2002; Briggs and Kolosov, 2010; Kundu, 2003):

$$
\begin{gathered}
R=\left|R\left(\alpha_{i}\right)\right|^{2} \\
T_{C}=\frac{\rho_{S} \tan \alpha_{i}}{\rho \tan \alpha_{C}}\left|T_{C}\left(\alpha_{i}\right)\right|^{2} \\
T_{S}=\frac{\rho_{S} \tan \alpha_{i}}{\rho \tan \alpha_{S}}\left|T_{S}\left(\alpha_{i}\right)\right|^{2}
\end{gathered}
$$

In the case where the angle of incidence is equal to zero only the compressional wave is transmitted in the sample. The energy of the reflection and transmission coefficients is given by (Briggs and Kolosov, 2010):

$$
\begin{aligned}
& R\left(0^{\circ}\right)=\left(\frac{Z_{C}-Z}{Z_{C}+Z}\right)^{2} \\
& T\left(0^{\circ}\right)=\frac{4 Z_{C} Z}{\left(Z_{C}+Z\right)^{2}}
\end{aligned}
$$

Note that, these coefficients are presented in this study in percentage, and the law of conservation of energy is always verified $(T+R=1)$.

This calculation procedure is performed using a program under the MATLAB software, which allows us to plot the energy curves of the transmission and reflection coefficients at the liquid/specimen interface as shown in Fig. 4.

\subsection{Strength measurement}

The mechanical tests are carried out at the age of 2, 7, 14 and 28 days, with a universal testing machine. First, the test pieces of cement paste $(4 \times 4 \times 16)$ $\mathrm{cm}^{3}$ are subjected to a three-point bending test, at the rate of three specimens for each series. The mechanical compression test is then performed on each halfprism with a section $(4 \times 4) \mathrm{cm}^{2}$ of the previously tested samples. The compressive strength is calculated by taking the average value obtained on 
six test pieces. In this study, the loading speed applied is equal to $0.5 \mathrm{MPa} / \mathrm{s}$. The results are summarized in Table 2.
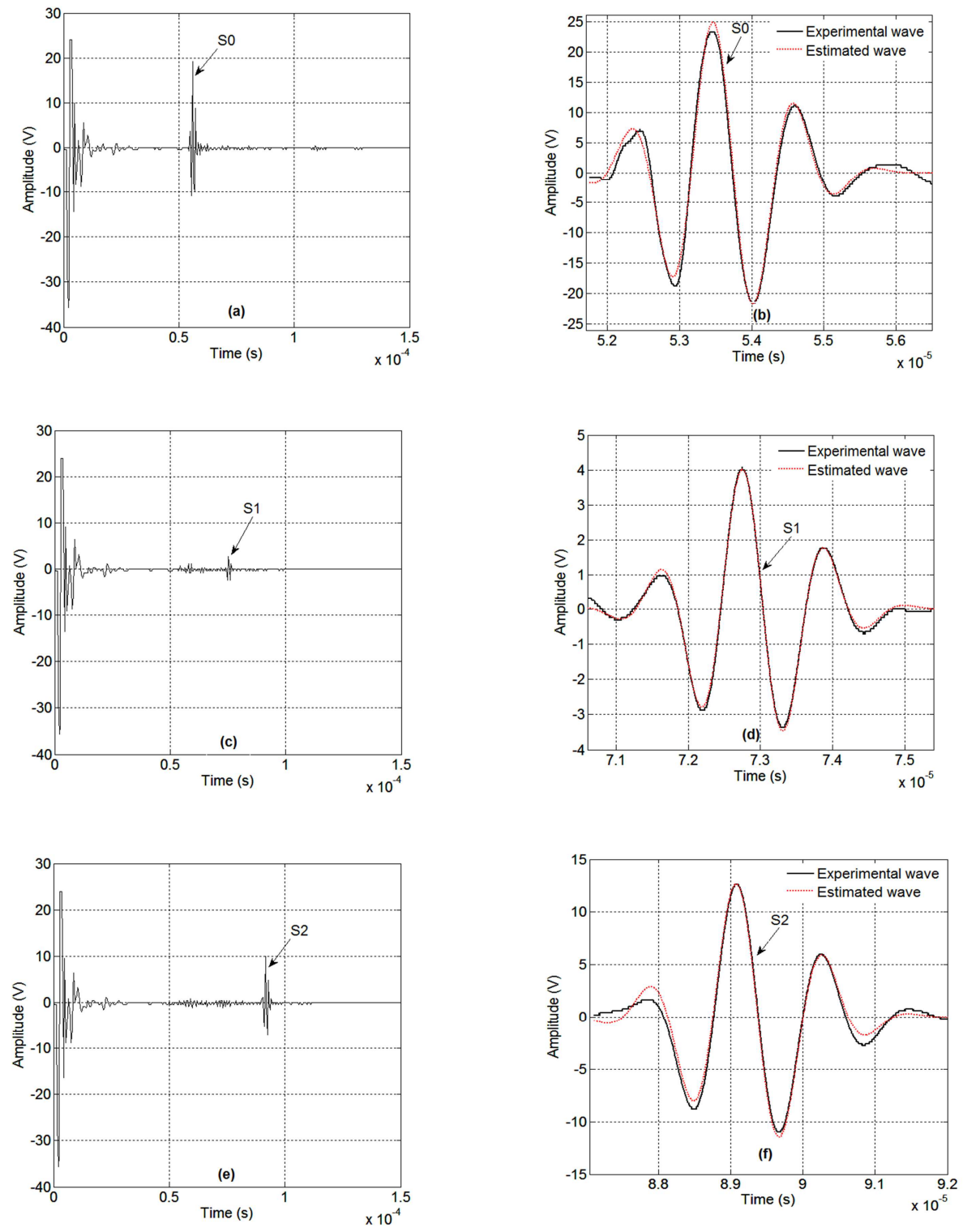

Fig. 3. Experimental (left) and simulated (right) echoes for cement paste with $w / c=0.55$ at $7^{\text {th }}$ day: a) b) Echo from the front face of the specimen, c) d) Echo related to $\mathrm{C}$-wave, e) f) Echo related to $\mathrm{S}$-wave 


\section{RESULTS AND DISCUSSION}

The results collected include two main parts. First, it will be presented the effect of the water-cement ratio and the curing time on the evolution of the wave velocity (compressional and shear), the coefficient (of reflection and transmission) and the compressive strength of cementitious samples. Then, correlations is presented between ultrasonic measurement results and compressive strength, the effect of each parameter was discussed by analyzing the results obtained experimentally.

\subsection{Evolution of the wave velocities in a white cement pastes}

Figure 5 shows the results of wave velocities in white cement pastes. The velocity of the compressional and shear waves is presented on the vertical axis while the horizontal axis designates the curing time. The storage conditions are kept constant in the water. As expected, the velocity increases with increasing curing time. However, there is a rapid increase of $4 \%$ on average up to the sixth day for $V_{C}$ and $3 \%$ for $V_{S}$. The increase continued with a slower pace from the seventh day to the fourteenth day, which is of the order of $0.55 \%$ for $V_{C}$ and $0.60 \%$ for $V_{S}$ (on average) and beyond the fourteenth day, a stabilization is noticed.This stabilization bearing for the following $w / c$ ratios: $0.35,0.40,0.45$, $0.50,0.55$ and 0.60 is fixed at a compressional velocity of the order of 5699.61, $5326.37,4934.43,4561.51,4299.19$ and $4130.05 m / s$, respectively. While the following bearings are recorded for the shear velocity 2985.49, 2774.23, $2557.22,2323.93,2202.94$ and $2065.33 \mathrm{~m} / \mathrm{s}$, respectively for the ratios $w / c$.

The difference between the $w / c$ ratios is relatively small, however the incrementation of the velocity is clearly observed. These curves show that $V_{C}$ and $V_{S}$ are inversely proportional to the $w / c$ ratios. Each 0.05 increase in this ratio gives us at 28 days a fall of $8.93,4.77,8.96,5.24,5.10 \%$ for $V_{C}$ and 7.47 , $6.32,9.35,5.35,7.06 \%$ for $V_{S}$, respectively cited. These results are not consistent with those obtained by Al-Akhras and Al-Qadi (1998) who stated that the watercement ratio has an insignificant effect on ultrasonic velocity. Indeed, the decrease in velocities is caused by the increase in porosity which is relative to the increase of the ratio $w / c$. This assumption is supported by Soltani recent work (Soltani, 2010).

\subsection{Development of reflection coefficient}

The intensity of the reflection coefficient is a function of density, velocity (of the water and the sample) and the angle of incidence. The increase in the ratio $w / c$ induces a decrease in the density and an increase in velocity. However, the rate of this increase is higher than the rate of decrease in density for that there has been a rise in the acoustic impedance. The difference in impedance between the 
two medium (liquid / solid) is so great that it always results in a positive reflection.

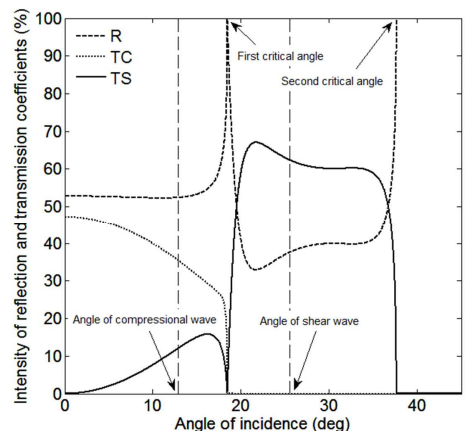

Fig. 4. Reflection and transmission coefficients for cement paste with $w / c=0.45$ at $7^{\text {th }}$ day
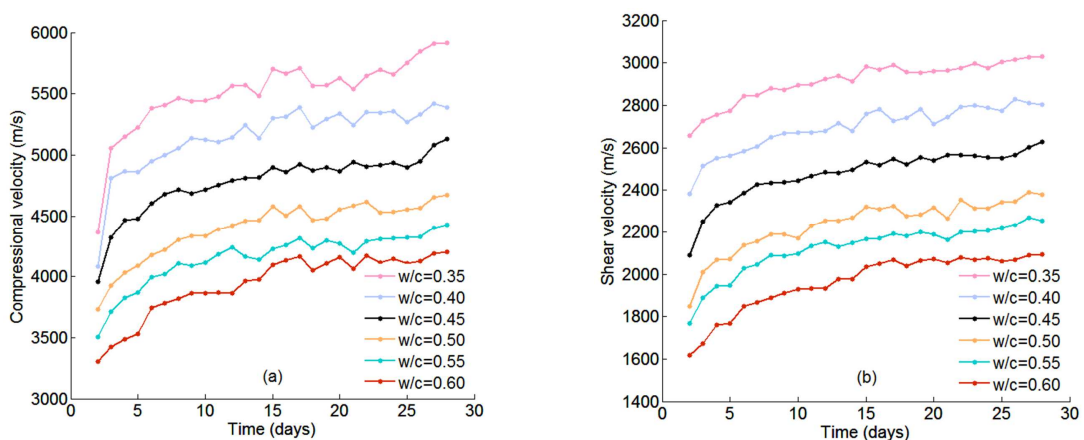

Fig. 5. The influence of $w / c$ during the curing time: a) The compressional wave velocity, b) The shear wave velocity
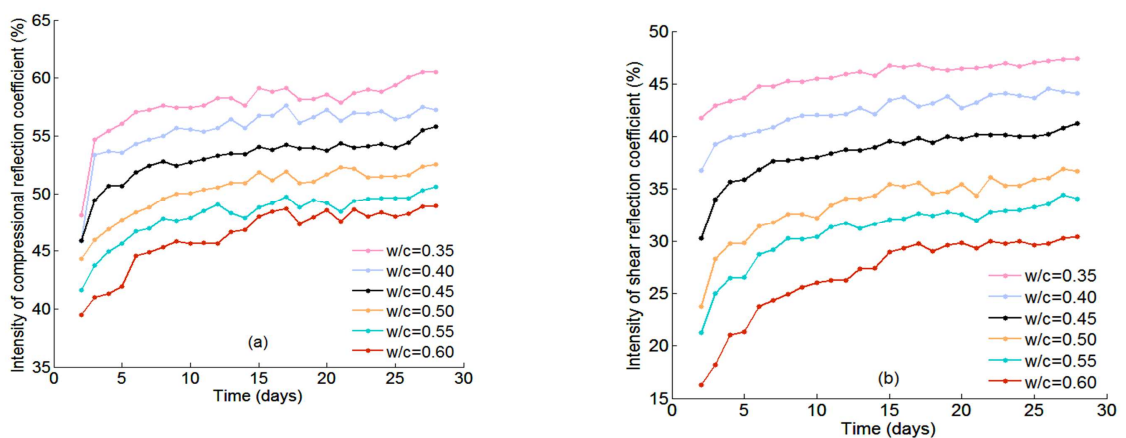

Fig. 6. Reflection coefficient development for white cement pastes: a) The compressional wave, b) The shear wave 
To compare the cementitious samples with the reflection coefficient, Fig. 6 is presented which illustrates the variation of the reflection coefficient intensity with $w / c$, for both compressional and shear waves. The curves of the reflection coefficient and the velocity have the same pace (behavior) depending on the day. The curves of the reflection coefficient and the velocity have the same shape (behavior) depending on the day. As can be seen, specimens that have a high ratio of $w / c$ have low reflection intensity. Thus, the results show a proportional increase as a function of the cure time, where there is a stabilization from the $14^{\text {th }}$ day which is visible in Fig. 6. These curves show that reflection measurements have the potential to distinguish between cementitious pastes. The variation band of the compressional reflection intensity $\left(R_{C}\right)$ is between $39 \%$ and $61 \%$, whereas for the shear mode $\left(R_{S}\right)$ is limited between $16 \%$ and $48 \%$.

From Fig. 7, these coefficients can be deduced at any oblique incidence angle and even the case of normal incidence.

\subsection{Variation of angles $\alpha_{1,2}$ and $\alpha_{c r} 1,2$}

In order to discuss the key angles of this method, the influence of the variation of $w / c$ ratio on these angles is presented in Table 1.

The angles of incidence $\left(\alpha_{1,2}\right)$ are positively proportional to the ratio $w / c$ and it was the case even for critical angles $\left(\alpha_{c r} 1,2\right)$, this is clearly visible in Fig. 7. This can be explained by the evolution of the microstructural state which is probably related to the porosity of the cementitious pastes. On the other hand, during the curing time these angles are almost stable for 28 days.

During the curing time and for all the cement samples tested, it has been found experimentally that there is an angle that allows visualization of both waves (compressional and shear) at the same time. The interval of this angle is between the angle of incidence $\alpha_{1}$ and the first critical angle $\left(\alpha_{c r 1}\right)$. This case study was treated by Merdjana et al. (2018) where they found that this angle is of the order of $13^{\circ}$ for the sample of cement paste.

Table 1. Incidence and critical angles of cement pastes

\begin{tabular}{|c|c|c|c|c|c|c|}
\hline $\boldsymbol{w} / \boldsymbol{c}(\mathbf{\%})$ & $\mathbf{0 . 3 5}$ & $\mathbf{0 . 4 0}$ & $\mathbf{0 . 4 5}$ & $\mathbf{0 . 5 0}$ & $\mathbf{0 . 5 5}$ & $\mathbf{0 . 6 0}$ \\
\hline $\boldsymbol{\alpha}_{\mathbf{1}}(\mathbf{d e g})$ & 10.21 & 11.23 & 11.80 & 12.98 & 13.71 & 14.46 \\
\hline $\boldsymbol{\alpha}_{\text {cr1 }}(\mathbf{d e g})$ & 14.52 & 15.98 & 16.81 & 18.52 & 19.58 & 20.68 \\
\hline $\boldsymbol{\alpha}_{\mathbf{2}}(\mathbf{d e g})$ & 20.25 & 21.97 & 23.54 & 26.14 & 27.74 & 30.05 \\
\hline $\boldsymbol{\alpha}_{\text {cr2 }}(\mathbf{d e g})$ & 29.31 & 31.94 & 34.39 & 38.53 & 41.16 & 45.09 \\
\hline
\end{tabular}




\subsection{Variation of the compressive strength}

Table 2 shows the variation in the compressive strength of the six different percentages $w / c$. Depending on the age and for each $w / c$ ratio, the compressive strength increases continuously until the 28th day. A reduction in strength is produced with each increase in the ratio $w / c$ ranging from $54.39 \mathrm{MPa}$ for $w / c=0.35$, up to $24.47 \mathrm{MPa}$ for a ratio of 0.60 . From the results, it is apparent that the ratio $w / c$ strongly affects the kinetics of evolution of the compressive strength.

\subsection{Relationship between $S$ and $V_{C, S}$}

Many works have been made on the study of the correlation between the UPV and strength of cementitious materials, several relationships have been proposed. According to previous studies, researchers often conclude exponential functions (Trtnik et al., 2009; Uddin and Mahmood, 2016).

$$
S=a e^{b V_{C, S}}
$$

Where $\mathrm{S}$ is the compressive strength (MPa), $a$ and $b$ are empirical constants.

Figure 8 shows the relationship between $S$ and $V_{C, S}$ of the cement pastes for all specimens of this study. From the experimental results, the following exponential equations have been proposed:

$$
\begin{aligned}
& S=1.499 e^{0.0006 V_{C}} \\
& S=1.895 e^{0.0011 V_{S}}
\end{aligned}
$$

These results show that there is a good correlation with a reasonable precision between the strength and velocity, where the coefficients $\mathrm{R}^{2}$ are acceptable with values of the order of 0.93 for the compressional velocity and 0.87 for the shear velocity.

\subsection{Relationship between $S$ and $\mathrm{R}_{\mathrm{C}, S}$}

An approach similar to the previous one $\left(S-V_{C, S}\right)$ was adopted in this part to find a relationship between the compressive strength and the reflection coefficient for all the studied mixtures. This relationship was among the objectives of this study. In fact, the exponential relationships shown in Fig. 9 have acceptable $R^{2}$ coefficients which are of the order of 0.95 for $\mathrm{R}_{\mathrm{C}}$ and 0.88 for $R_{S}$.

$$
\begin{aligned}
& S=0.3288 e^{0.0852 R_{C}} \\
& S=3.3040 e^{0.0573 R_{S}}
\end{aligned}
$$


These relationships can be exploited to calculate the strength of cementitious pastes using the coefficient of reflection coefficient with adequate precision.

Table 2. Evolution of compressive strength, Mpa

\begin{tabular}{|c|c|c|c|c|c|c|}
\hline $\boldsymbol{\rho}\left(\mathbf{k g} / \mathbf{m}^{3}\right)$ & $\begin{array}{c}w / c=0.35 \\
\rho=1907\end{array}$ & $\begin{array}{c}w / c=0.40 \\
\rho=1861\end{array}$ & $\begin{array}{c}w / c=0.45 \\
\rho=1805\end{array}$ & $\begin{array}{c}w / c=0.50 \\
\rho=1787\end{array}$ & $\begin{array}{c}w / c=0.55 \\
\rho=1731\end{array}$ & $\begin{array}{c}w / c=0.60 \\
\rho=1712\end{array}$ \\
\hline $\mathbf{0 2}$ days & 21.21 & 19.64 & 17.71 & 11.13 & 08.87 & 07.88 \\
\hline $\mathbf{0 5}$ days & 36.50 & 29.46 & 23.52 & 16.74 & 14.25 & 12.67 \\
\hline $\mathbf{0 7}$ days & 39.40 & 30.29 & 26.78 & 17.79 & 15.90 & 13.17 \\
\hline $\mathbf{1 4}$ days & 44.31 & 36.12 & 33.24 & 25.82 & 21.44 & 16.43 \\
\hline 21 days & 48.08 & 39.95 & 37.88 & 27.82 & 24.83 & 18.55 \\
\hline 28 days & 54.39 & 49.98 & 41.11 & 30.57 & 28.04 & 24.47 \\
\hline
\end{tabular}

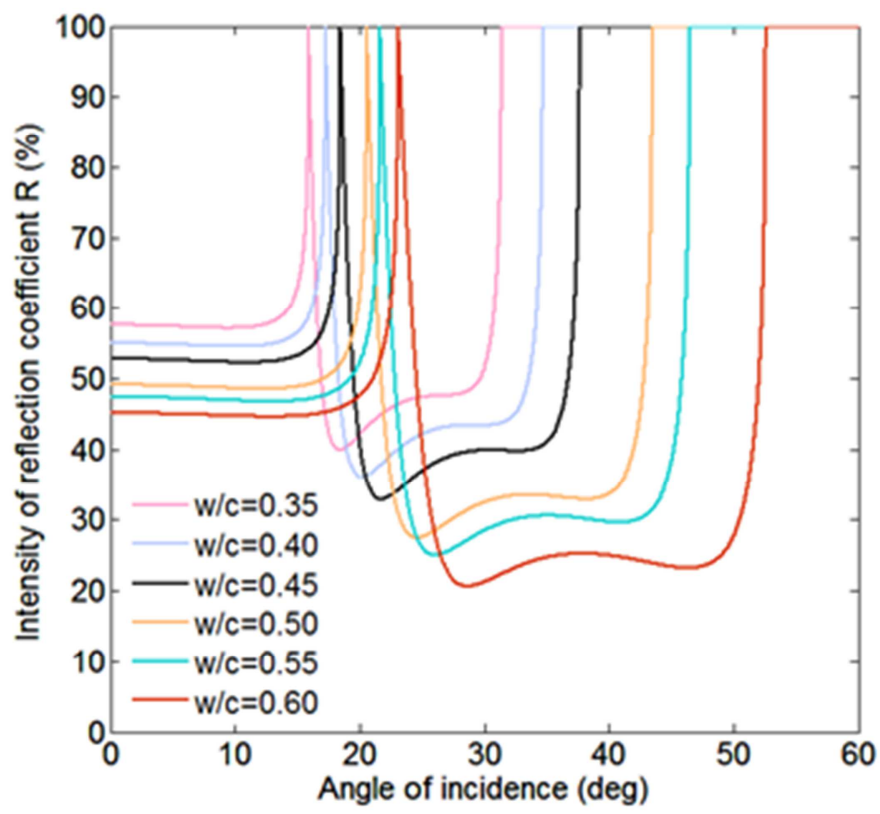

Fig. 7. Reflection coefficient for different cement pastes depending on angle of incidence 

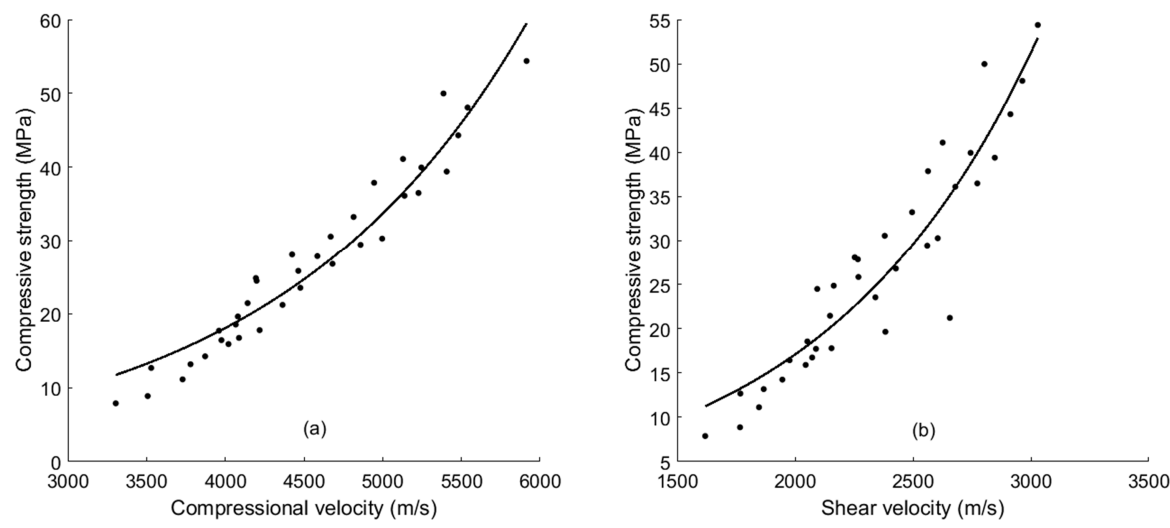

Fig. 8. Relationship between compressive strength and the velocity: a) The compressional velocity, b) The shear velocity
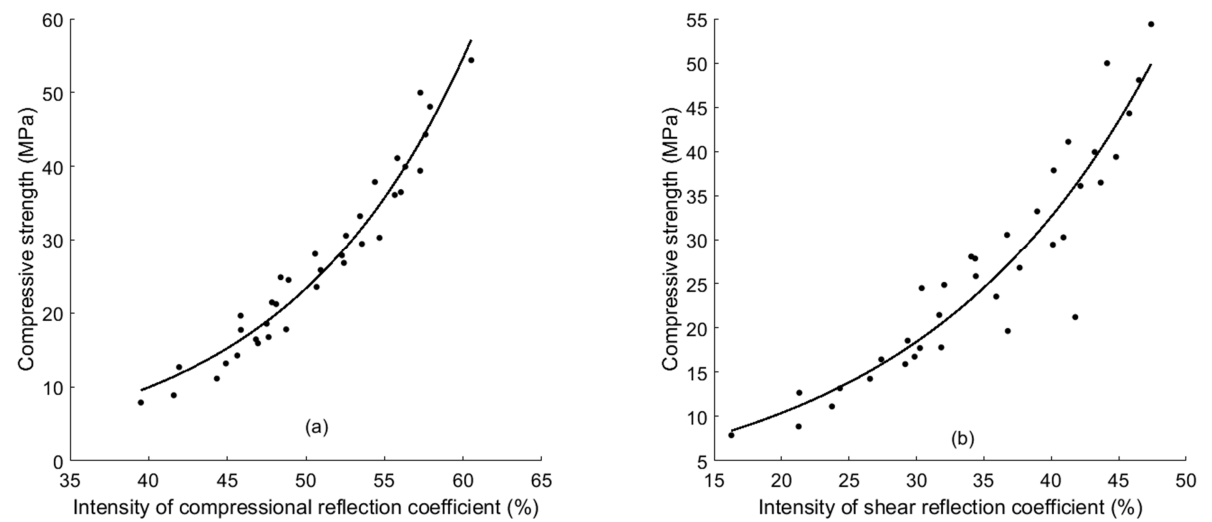

Fig. 9. Relationship between compressive strength and the reflection coefficient: a) The compressional reflection coefficient, b) The shear reflection coefficient

\section{CONCLUSIONS}

The initiative taken in this study aims to establish a substitution of the conventional relationship between velocity and compressive strength by replacing the velocity by the reflection coefficient during the curing time. The particularity of the prism technique lies in the potential for obtaining compression and shear wave velocities using specific incidence angles. These parameters can be obtained from a single test. This experiment was carried out 
on specimens of cement paste with a variation of the ratio $w / c$. The experimental results allow us to draw the following conclusions:

- The velocity and the reflection coefficient are both affected by the $w / c$ ratio. They have the same relative variation as a function of days and are distinguished by an inversely proportional relationship with $w / c$.

- Critical and incidence angles are indicator parameters for the distinction of materials.

- The relationship between velocity and the reflection coefficient with the compressive strength are exponential with good correlations where the values of the coefficient $R^{2}$ are between 0.87 and 0.95 .

The contribution of this study provides a new simplified expression to predict the strength of cementitious materials by the reflection coefficient using the prism technique. The approach carried out allows a better understanding of the utility of this technique. In addition, the device has the advantage of being flexible and scalable according to the required conditions and has a great ability of adaptation to characterize different types of materials.

\section{REFERENCES}

1. Abo-Qudais, SA 2005. Effect of concrete mixing parameters on propagation of ultrasonic waves. Construction and Building Materials 19, 257-263.

2. Aggelis, DG, De Sutter, S, Verbruggen, S, Tsangouri, E and Tysmans, T 2019. Acoustic emission characterization of damage sources of lightweight hybrid concrete beams. Engineering Fracture Mechanics 210, 181-188.

3. AI-Akhras, NM and AI-Qadi, IL 1998. Using ultrasonic waves to detect basic properties of Portland cement concrete. The Indian Concrete Journal, 72 (1), 33-37.

4. Al-Mufti, RL and Fried, AN 2012. The early age non-destructive testing of concrete made with recycled concrete aggregate. Construction and Building Materials 37, 379-386.

5. Blitz, J 1997. Electrical and magnetic methods of non-destructive testing, Second Edition, Springer Netherlands.

6. Bouhadjera, A and Bouzrira, C 2005. High-frequency ultrasonic testing of young cement-based materials using the 'prism technique'. NDT\&E International 38, 135-142.

7. Boumiz, A, Vernet, C and CohenTenoudji, F 1996. Mechanical properties of cement pastes and mortars at early ages: Evolution with time and degree of hydration. Advanced Cement Based Materials 3 (3-4), 94-106.

8. Briggs, GAD and Kolosov, OV 2010. Acoustic microscopy, Second Edition, Oxford University Press. 
9. Brozovsky, J 2014. High-strength concrete - NDT with rebound hammer: influence of aggregate on test results. Nondestructive Testing and Evaluation 29(3), 255-268.

10. David, J and Cheeke, N 2002. Fundamental and applications of ultrasonic waves, Second Edition, CRC Press New York, USA.

11. Dinh, K, Gucunski, N and Zayed, T 2019. Automated visualization of concrete bridge deck condition from GPR data. NDT \& E International 102, $120-128$.

12. Grimes, M, Bouhadjera, A, Haddad, S and Benkedidah, T 2012. In vitro estimation of fast and slow wave parameters of thin trabecular bone using space-alternating generalized expectation-maximization algorithm. Ultrasonics 52, 614-621.

13. Hellier, C 2003. Handbook of nondestructive evaluation, Second Edition, McGraw-Hill Education, USA.

14. Hermida, G 2008. Influence of paste volume and cement content upon concrete performance: toward the development of concrete with minimized paste, Ph.D. Cachan ENS.

15. Hernandez, MG, Izquierdo, MAG, Ibanez, A, Anaya, JJ and Ullate, LG 2000. Porosity estimation of concrete by ultrasonic NDE. Ultrasonics 38, 531-533.

16. Hussain, A and Akhtar, S 2017. Review of Non-Destructive Tests for Evaluation of Historic Masonry and Concrete Structures. Arabian Journal for Science and Engineering 42(3), 925-940.

17. Kheder, GF, Al Gabban, AM and Abid, SM 2003. Mathematical model for the prediction of cement compressive strength at the ages of 7 and 28 days within 24 hours. Materials and Structures 36, 693-701.

18. Krauträmer, H and Krauträmer, J 1990. Ultrasonic testing of materials, 4rd Edition, Springer-Verlag: Berlin, Heidelberg, New York.

19. Kundu, T 2003. Ultrasonic nondestructive evaluation: Engineering and Biological Material Characterization, CRC Press London.

20. Merdjana, H, Boukabou, A and Grimes, M 2018. A high accuracy ultrasonic measurement system using the prism technique. Measurement 114, 195-202.

21. Mix, PE 2005. Introduction to nondestructive testing: A training guide, Second Edition, John Wiley \& Sons, USA.

22. Panzera, TH, Rubio, JC, Bowen CR, Vasconcelos, WL and Strecker, K 2008. Correlation between structure and pulse velocity of cementitious composites. Advances in Cement Research 20 (3), 101-108.

23. Payan, C, Abraham, O and Garnier, V 2018. Ultrasonic methods, Nondestructive Testing and Evaluation of Civil Engineering Structures, 21-85. 
24. Qasrawi, HY 2000. Concrete strength by combined nondestructive methods simply and reliably predicted. Cement and Concrete Research 30, 739-746.

25. Soltani, F 2010. Characterization of cement paste by ultrasonic methods, Ph.D. EC-Lille.

26. Trtnik, G, Kavčič and F, Turk, G 2009. Prediction of concrete strength using ultrasonic pulse velocity and artificial neural networks. Ultrasonics 49, 53-60.

27. Turgut, $\mathrm{P}$ and Kucuk, OF 2006. Comparative relationships of direct, indirect, and semi-direct ultrasonic pulse velocity measurements in concrete. Russian Journal of Nondestructive Testing 42 (11), 745-751.

28. Uddin, MT and Mahmood, AH 2016. Effects of maximum aggregate size on UPV of brick aggregate concrete. Ultrasonics 69, 129-136.

29. Voigt, T 2005. The Application of an ultrasonic shear wave reflection method for nondestructive testing of cement-based materials at early ages: An experimental and numerical analysis, University of Leipzig, Germany, Books on Demand.

Editor received the manuscript: 19.12.2019 